\title{
La cooperativa de segundo grado: perspectivas para su desarrollo en Cuba
}

\author{
(Second grade cooperatives: perspectives for their \\ development in Cuba) \\ Nileidys Torga Hernández ${ }^{1}$ \\ Orestes Rodríguez Musa² \\ Universidad de Pinar del Río
}

Sumario: I. Introducción. II. La cooperativa de segundo grado: tras un punto de partida. III. La cooperativa de segundo grado en Cuba: antecedentes, actualidad y perspectivas. 3.1. Antecedentes. 3.2. Contexto actual y perspectivas. IV. Conclusiones. V. Bibliografía

Resumen: El proceso de actualización del modelo socioeconómico cubano incluye, entre otras transformaciones, el fortalecimiento del sector cooperativo, tanto que se prevé avanzar hacia la constitución de cooperativa de segundo grado. En tal sentido, el objetivo de este trabajo es valorar las actuales condiciones socioeconómicas y jurídicas sobre las que se proyecta la instrumentación de cooperativas de segundo grado en Cuba. Para ello, se comenzará sentando una base teórica en torno a esta figura. En un segundo momento se indagará en las experimentales experiencias de este tip. de cooperativas que han existido en el país. Por último, se analizarán las limitaciones de las recién constituidas cooperativas no agropecuarias en Cuba, a partir de las que se pretende avanzar en la integración cooperativa.

1 Profesora de Derecho de Obligaciones y Derecho de Contratos en el Departamento de Derecho de la Facultad de Ciencias Sociales y Humanísticas de la Universidad de Pinar del Río, Cuba. Licenciada en Derecho por la Facultad de Derecho de la Universidad de la Habana (2005), Máster en Derecho de Familia por la propia institución (2011) y Licenciada en Economía por la Facultad de Economía y Contabilidad de la Universidad de Pinar del Río (2013). Correo electrónico: nileidys@fcsh.upr.edu.cu

2 Profesor de Derecho Constitucional y de Introducción al Derecho de Cooperativas en el Departamento de Derecho de la Facultad de Ciencias Sociales y Humanísticas de la Universidad de Pinar del Río, Cuba. Licenciado en Derecho por la Facultad de Derecho de la Universidad de la Habana (2006) y Máster en Derecho Constitucional y Administrativo por la propia institución (2010). Investigador del Centro de Estudios de Desarrollo Cooperativo y Comunitario (CEDECOM) adscrito a la Universidad de Pinar del Río, Cuba e investigador de la Asociación Internacional de Derecho Cooperativo (AIDC) con sede en la Universidad de Deusto, España. Correo electrónico: musa@upr.edu.cu 
Palabras clave: cooperativas de segundo grado.

Abstract: The process of upgrade of the socioeconomic Cuban pattern includes, among other transformations, the invigoration of the cooperative sector. That includes to advance toward the constitutional regulation of the cooperative of second grade. In that order, the objective of this work is to value the current socioeconomic and juridical conditions to project the instrumentation of cooperative of second grade in Cuba. For that matter, we will begin sitting down a theoretical bases around this figure. In a second moment we will research in the experimental experiences of this typ. that existed in the country. Lastly, we will analyse the recently experimental cooperatives constituted on the not agricultural sector in Cuba.

Key words: second grade cooperatives. 


\section{Introducción}

La tendencia a la globalización, la crisis económica y la hostilidad del mercado se erigen como el espacio propicio para que proliferen formas empresariales más competitivas que garanticen la eficiencia económica. Ante esta realidad las cooperativas, para preservar su identidad, continúan obligadas a crecer, aprovechando las ventajas de fórmulas superiores de integración.

Entre los principales elementos que caracterizan el contexto cubano actual se halla el proceso de actualización del modelo económico, que se ha reconocido como la alternativa para sustentar la construcción del socialismo en Cuba. Dicho proceso está regido por los Lineamientos de la Política Económica y Social del Partido y la Revolución, aprobados en el pleno del VI Congreso del Partido Comunista de Cuba. Un acercamiento al contenido de estos Lineamientos, nos permite advertir que la cooperativización jugará un papel importante en el escenario socioeconómico cubano de los próximos años.

En apretadísima síntesis pudieran definirse tres grandes transformaciones en el proceso de cooperativización al que se encuentra abocado nuestro país: la extensión de las cooperativas al sector no agropecuario, la consolidación de las cooperativas agropecuarias, y la posterior constitución de cooperativas de segundo grado.

La primera y segunda se manifiestan de manera más adelantada, lo cual no es casual sino consecuencia lógica del propio proceso y sus peculiaridades. Pero el terreno de las cooperativas de segundo grado en Cuba — donde se centra esta investigación - es prácticamente virgen. Ello no significa que el tema se encontrara fuera del camp. de visualización de los investigadores del cooperativismo, sino que estos han debido concentrar sus esfuerzos en perfeccionar su funcionamiento en el sector agropecuario y en su diversificación hacia otros sectores de la economía, antes de pretender ascender a peldaños superiores de integración del movimiento.

Habiéndose avanzado en este sentido, el objetivo de este trabajo es valorar las actuales condiciones socioeconómicas y jurídicas en que se proyecta política y legalmente la instrumentación de las cooperativas de segundo grado en Cuba. Para ello se comenzará sentando una necesaria base teórica en torno a algunas de las peculiaridades de esta figura. En un segundo momento se indagará en las experimentales experiencias de cooperativas agropecuarias de segundo grado en Cuba, como antecedentes a tener en cuenta. Por último, se analizarán las limitaciones de las recién constituidas cooperativas no agropecuarias, a partir de las cuales se proyecta avanzar en la creación de cooperativas de segundo grado. 


\section{La cooperativa de segundo grado: tras un punto de partida}

Las cooperativas de segundo o ulterior grado constituyen el tip. legal de cooperativas en torno al cual se organizan la mayor part. de los procesos de integración cooperativa.

Para BRUQue \& Moyano (2006) la sociedad cooperativa de segundo grado es «...una sociedad de las empresas agrupadas donde se forma la voluntad del grup. mediante la cooperación de todas las entidades agrupadas» (p.24). Para MARTínez (1990) «las cooperativas de segundo grado son aquellas formadas por otras de primer grado que son sus socios (...) En ellas se prolongan las ideas y bases de la cooperativa...» (p.43). Alfonso (2000) por su parte, viene a profundizar en la naturaleza jurídica de esta figura, al definir a las cooperativas de segundo grado como "cooperativa de cooperativas» y señalar que esta «... puede ser considerada como exponente de un grup. por coordinación de carácter externo y personificado» (p.270).

Las definiciones expuestas son escuetas, pero mencionan el principal elemento que distingue a las cooperativas de segundo grado: la agrupación de otras cooperativas. Además, coinciden en expresar que las cooperativas de segundo grado mantiene la forma organizativa de una cooperativa, en tanto las cooperativas de segundo grado — en líneas generales - mantienen las mismas características que las cooperativas de base y en su funcionamiento se rigen por los mismos principios.

A tono con su naturaleza, las cooperativas de segundo grado también deben estar dirigidas a desarrollar las ideas y fines de las cooperativas que la integran. Si bien no es unánime la aceptación de este elemento como fundamental, para los autores de este trabajo resulta básico a la hora de delimitarla ya que nos indica el porqué de su existencia, con independencia de la probable heterogeneidad de los miembros que la componen.

En cuanto a sus socios, la regla es que sean cooperativas, pero también se admite que ostenten la categoría de socios otras personas físicas o jurídicas. Al respecto se pronuncia PUENTES (2010) al ofrecer su concepto de cooperativas de segundo grado:

«Podemos definir de forma general a la sociedad cooperativa de segundo grado como una asociación de, al menos dos sociedades cooperativas de primer grado y de personas jurídicas, públicas o privadas - bajo unos límites establecidos en las leyes cooperativasque se agrupan con el objetivo de poder desarrollar en común una determinada actividad económica o empresarial que les permitan actuar de forma más competitiva en el mercado. (p. 53)» 
En efecto, aunque una de las notas definitorias de estas entidades sea la naturaleza cooperativa de sus socios, algunas legislaciones permiten que formen part. de la misma otros tipos de personas físicas o jurídicas que no encuadren en la categoría de cooperativas.

La cooperativa de segundo grado puede erigirse como cabecera de un grup. empresarial. Si comparamos la integración cooperativa con cualquier otro tip. de integración empresarial, podemos afirmar que coinciden en la creación de un grupo, pero con la particularidad diferenciadora de que se trata de un grup. por coordinación horizontal. En este caso, ninguna de las partes agrupadas toma el poder como ocurre en un grup. capitalista en el que una empresa funciona como cabeza dominante del grupo, sino que las empresas partícipes de dicho grup. coordinan, completan y desarrollan su actividad.

Sin embargo, la creación de una cooperativa de segundo grado no tiene que conducir necesariamente al nacimiento de un grup. empresarial por coordinación, este tema se caracteriza por su flexibilidad. Como indica Alfonso (2000) «...tan pronto puede ser una simple sociedad cooperativa como servir de vestidura jurídica a un grup. de sociedades cooperativas, caracterizado, en tal caso, por su alto nivel de integración» (p.269). La actuación o no como grup. cooperativo dependerá de cuál sea la finalidad de la misma y lo recogido en los estatutos en cuanto a la toma de decisiones y a la dirección ejercida dentro de la misma. Dependiendo del objeto social con el que se constituya, se definirá como un instrumento de colaboración entre cooperativas (intercooperación) o como un instrumento de integración empresarial.

En lo que sí existe consenso es en que las cooperativas de segundo grado poseen personalidad jurídica independiente de las cooperativas que la forman. Su constitución es consustancial a la creación de un ente jurídico distinto a los existentes, cuyo funcionamiento queda regulado por su estatuto, el cual puede contener normas distintas a las expresadas en los estatutos de las cooperativas socios (AIZEGA y VALIÑANI, 2003).

A pesar de las varias definiciones y posturas que podemos encontrar de cooperativas de segundo grado, nos parece ajustada la siguiente:

«La sociedad cooperativa de segundo grado es una sociedad cooperativa formada tanto por sociedades cooperativas de primer grado como por personas físicas o jurídicas y, con personalidad jurídica propia; cuyo propósito es el de desarrollar las ideas y los fines de las que la forman, y que con tal objetivo pasa a ser cabeza de un grup. de sociedades por coordinación. (GARrido, Puentes \& NAVARRO, 2007, p. 32).» 
De modo que independientemente del concepto más o menos enriquecido que quiera formularse, hay una serie de elementos que no deben dejar de decirse al definir las cooperativas de segundo grado. En primer lugar, aunque con características peculiares, estamos ante una entidad que tiene naturaleza cooperativa, con independencia de que sus socios pueden ser personas naturales, cooperativas de primer grado $u$ otras personas jurídicas con naturaleza distinta a la de una cooperativa; en segundo lugar constituyen una persona jurídica nueva, que ostenta personalidad jurídica propia e independiente de sus socios; por último, su surgimiento responde a la necesidad de desarrollo de sus miembros.

A tono con el último elemento señalado, vale destacar que diversas han sido las causas que han llevado a las cooperativas a emprender procesos de integración y muchos los autores que han tratado de sistematizarlas (MARTínez 1990; MONTERO, 1991 y 1999; SANCHÍS, 1995; Vargas, 1995; Arcas, Munera \& Hernández, 2002; Senise, 2003 y BruQUE \& MoYANO, 2006). Sin dejar de reconocer que la causa principal es la de crecer para sobrevivir, las razones colaterales más comunes pueden subdividirse en cuestiones de tip. económico, consecuencia de los cambios en el entorno económico, y razones de tip. social, asociadas a la propia naturaleza especial de estas entidades. Las primeras, también denominadas genéricas o externas, se refieren a la necesidad de adaptarse al contexto y a los mercados, buscando un mayor tamaño que les permita actuar con más eficiencia. Las segundas, reconocidas como específicas o internas, se relacionan con el desarrollo del movimiento cooperativo que lleva necesariamente a la intercooperación.

Según Puentes, Antequera \& Velazco (2010) estas causas se resumen en:

- Razones económicas: necesidad de ser competitivos, tras la globalización y la liberalización de las economías; tendencia a la concentración empresarial del resto de sociedades; acceso a las nuevas tecnologías de la información y comunicación; y obtención de ventajas que conlleva un mayor tamaño en la empresa (economías de escala, acceso a fuentes de financiación, mayor poder de mercado e incremento del poder frente la competencia).

- Razones sociales: defensa del movimiento cooperativo; apoyo a las sociedades cooperativas de base y apoyo a las causas sociales.

Vale acotar que aun cuando la integración del cooperativismo no fuera resultado de la necesidad de supervivencia en una economía competitiva, la propia definición de empresa cooperativa nace ya como «...embrión concentrador de esfuerzos dispersos», tal como apunta PA- 
RRAS (1974, p.4). De hecho, el propio principio cooperativo de «cooperación entre cooperativas» ya justifica este fenómeno (MARTínEZ, 2012).

Las ventajas de la constitución de cooperativas de segundo grado han sido estudiadas por diversos autores (VARGAS, 1995; MONTERO, 1999 y ARCAS, MUNERA \& HERNÁNDEZ, 2002) y los análisis se concentran fundamentalmente en los ámbitos de la organización y la comercialización. Entre las ventajas más significativas podemos destacar, siguiendo a Puentes, Antequera y Velazco (2010):

- La sociedad cooperativa de segundo grado es un instrumento de integración que permite no obviar los principios cooperativos, con lo cual las sociedades agrupadas mantendrán su origen cooperativo, sin necesidad de adaptarse a nuevas formas de organización y funcionamiento. Las sociedades que se integran no desaparecen y siguen manteniendo su autonomía jurídica y empresarial respecto a la primera, no surgiendo así ningún tip. de reticencia a la constitución de la misma por part. de las sociedades cooperativas de base.

- Al no existir relaciones de dominio y las decisiones tomarse por acuerdos paritarios y libres, permite que las sociedades que se agrupen determinen el grado de implicación que quieren tener con el resto del grupo.

- Reportan una serie de beneficios a las sociedades cooperativas que la integran, en aspectos tales como administración, producción y comercialización.

- Disminuir costos, que entrañaría la puesta en marcha de una sociedad cooperativa de segundo grado, en los términos anteriormente expuestos. En este sentido amplía Vargas (1995, p. 149) identificando los siguientes aspectos: centralización, en la entidad de segundo grado de la gestión empresarial de las sociedades cooperativas de base, lo que permitirá una reducción de costos de gestión; acceso a ayudas públicas para inversiones que de forma individual no se podrían realizar (mejora de instalaciones de transformación y comercialización, etc.).

Sobre algunos de estos extremos se pronuncian también otros autores como Melí́ (2003), quién señala como aspecto positivo «...la posibilidad que les ofrece esta fórmula de conservar su independencia y su personalidad jurídica, permitiéndoles elegir un mayor o menor grado de compromiso con respecto al resto de las entidades» (p. 8).

Por su part. Mozas y Bernal (2004, p.145) señalan que «han permitido una mayor concentración de la oferta, la diversificación de productos y calendarios, la apertura de nuevos mercados, la contratación 
de servicios y adquisición de inputs de forma conjunta, la adopción de nuevos procesos industriales y la proliferación de la gestión».

En torno a la integración de las cooperativas agrarias concretamente, apunta ARCAS (2002) que las cooperativas de segundo grado contribuyen a que las que la integran se orienten al mercado y consigan sus objetivos empresariales.

Pese a que - como hemos podido observar - son numerosas las ventajas de la integración, es necesario reconocer que esta no está exenta de dificultades. Las ventajas de la constitución de cooperativas de segundo grado se aderezan con algunas desventajas que se les reconoce al proceso.

Tal y como señala VARGAS (1995) la participación en una cooperativa de segundo o ulterior grado «...tiene como contrapartida para las cooperativas que la integran la pérdida de capacidad de decisión a favor de la unidad de acción, debiendo acatar y cumplir con lo acordado en los órganos superiores de decisión» (p. 138). Por este motivo las cooperativas deben de valorar si están dispuestas a ceder poder de decisión, manteniendo su independencia económica, hacia la unidad de grado superior, a cambio de los beneficios o ventajas que su integración le reportará más a largo plazo.

Por su part. MELIÁ (2003) afirma que:

«... el hecho de implicar el mantenimiento en muchos casos de varias estructuras productivas, a la vez que administrativas, comerciales, etc., conlleva que soporten unos costes por fuerza superiores a los que asumen las cooperativas que han optado por fórmulas de concentración con vinculación patrimonial, tales como la fusión. (p. 8).»

Quizás con mayor preocupación por la naturaleza cooperativa, PARRAS (1974), MARTínez (1990) y MoReno (2001) señalan que las principales dificultades a las que se enfrentan las cooperativas de segundo grado se sintetizan en:

- Adopción de una democracia delegada. Nos referimos, a que las sociedades cooperativas de base han de actuar en las sociedades cooperativas de segundo grado a través de representantes elegidos democráticamente. Esta situación puede conllevar un desinterés por part. de los socios al no verse involucrados directamente en la de grado superior.

- Capacitación técnica de los directivos. En muchos casos las estructuras de segundo grado no disponen de personal cualificado para una gestión eficaz y eficiente de este tip. social, lo que dificulta el desarrollo e implantación de las mismas. 
Además de estas dos dificultades no podemos olvidar la presencia del elemento subjetivo, que por lo general ofrece resistencia al cambio. Como señala VARGAS (1995) «...aunque parezca paradójico, quizá el principal problema para poner en marcha la estrategia integradora venga del lado de los más interesados en el proyecto: los propios socios». (p.41)

En este sentido vuelve a operar y cobrar importancia el principio cooperativo de «educación, capacitación e información». Los socios necesitan ser informados de las ventajas que suponen para ellos el pertenecer a una cooperativa de grado superior, y se debe fomentar su participación en la toma de decisiones. El éxito de este proceso se encuentra íntimamente relacionado con la capacitación de los implicados y las circunstancias que los rodean pues sin su voluntad y preparación difícilmente tenga éxito la integración.

\section{Las cooperativas de segundo grado en Cuba: antecedentes, actualidad y perspectivas}

En Cuba, la Asociación Nacional de Agricultores Pequeños (ANAP) ha desempeñado funciones que algunos han equiparado a las de una federación de cooperativas agropecuarias. Esta labor de coordinación en favor del sector cooperativo agropecuario nacional, que también ha tenido como objetivo mantener la unidad ideológica del movimiento, no es criterio de los autores que deba confundirse con una estructura cooperativa-empresarial de grado superior. Sin embargo, es de reconocer que en algún momento favoreció experiencias de este tip. en el sector agropecuario de la economía, que a continuación estudiaremos siguiendo a CRUZ (2012).

\section{III.1. Antecedentes}

La génesis de este tip. de organización funcionó en Cuba para la comercialización de insumos en 1982 con el nombre de Unión Municipal de Cooperativas Agropecuarias Experimental (UMCA-E). Esta surgió al calor de las acciones emprendidas por el Estado cubano en aras de incrementar las formas de prestar apoyo y asistencia a toda la producción campesina, después del I Congreso del Partido Comunista de Cuba.

En el contexto de las búsquedas de las formas y vías para incrementar la producción y mejorar los abastecimientos del sector campesino, en la reunión del Buró Político del Comité Central del Partido Comu- 
nista de Cuba de agosto de 1981, fue aprobado organizar, con carácter experimental, cooperativas de comercialización de insumos para el sector campesino en algunos municipios elegidos en diferentes lugares del país.

Las Uniones Municipales de Cooperativas que se crearon -experimentalmente- perseguían sustituir y perfeccionar las estructuras de dirección estatal que manifestaban ineficiencia en su gestión. La forma de actuación sería a nivel municipal como la de una empresa independiente con personalidad jurídica propia.

La Asociación Nacional de Agricultores Pequeños (ANAP) asumió la responsabilidad de apoyar y acompañar el proceso de organización de esas cooperativas de comercialización. En coordinación con el Ministerio de la Agricultura (MINAGRI) se procedió a seleccionar municipios de diversos tamaños para llevar a cabo el experimento. La primera UMCA-E creada en el país comenzó a funcionar en San Antonio de los Baños en marzo de 1982.

La nueva organización económica absorbió a la Dirección de Atención al Sector Campesino (DASC) y las Empresas de Aseguramientos al Sector Campesino (EASC) y se planteó como objetivos:

- Mejorar en la organización de los abastecimientos de insumos y servicios productivos a las cooperativas y campesinos individuales.

- Elevar la efectividad de los métodos de dirección para dar respuestas a los problemas que tiene el sector no estatal.

- Fortalecer las relaciones de la unión con las instituciones del gobierno local, con el banco y otras empresas.

- Reducir los volúmenes de pérdidas con que trabajaban las EASC. La producción cañera fue excluida de su esfera de acción.

- Desagregar el plan productivo en cada municipio entre los actores sociales correspondientes.

La constitución de las UMCA-E estuvo precedida de un examen de viabilidad económica. Para su creación se exigieron como requisitos:

- Aprobación y apoyo de su funcionamiento por las cooperativas y campesinos de los territorios correspondientes.

- Autonomía de gestión.

- Reconocimiento de su personalidad jurídica por cualesquiera otras empresas e instituciones dentro y fuera del territorio de sus operaciones.

- Disfrute del descuento comercial para todos los productos planificados que suministrase a los productores.

— Funcionamiento con bajo nivel de rentabilidad. 
Los campesinos de los territorios donde se crearon las UMCA aportaron a estas un préstamo, como financiamiento inicial para comenzar operaciones. Ellas no disponían de capital inicial por lo cual se vieron obligadas a pagar inmediatamente los medios básicos que obtuvieron.

Entre las ventajas que significó la UMCA se resaltan

- Buscaron en la propia localidad la fuente de los ahorros materiales, monetarios y laborales.

- La presencia permanente de los mejores especialistas en las áreas productivas permitía a la unión desarrollar un intenso asesoramiento a los productores.

- El hecho de que dispusieran de la facultad de emitir órdenes de cobro y pago y de establecer relaciones económicas con diversas empresas suministradoras facilitaba su gestión.

- Las estrechas relaciones con los Bancos para el uso, control y devolución de los medios financieros prestados, favoreció la reducción de los créditos vencidos y prorrogados.

- Su colaboración fue decisiva en la satisfacción de las necesidades productivas de las cooperativas.

- El funcionamiento se caracterizó por la democracia, el alto nivel de gestión y la búsqueda de racionalidad económica.

- Establecimiento de relaciones más directas, transparentes y participativas entre con los cooperativistas.

- Actuaron como elemento de la promoción de la colaboración, de generalizador de las mejores experiencias productivas y no como entidad colocada por encima de los productores.

En análisis de la experiencia de las UMCA-E presentados por la Dirección Nacional de la ANAP en febrero de 1985 y datos suministrados por el Centro de Estudios de la Economía Cubana en 1987, se demostraron crecimientos productivos en los territorios de referencia ${ }^{3}$ y se ejecutaron centenares de obras sociales.

En realidad una part. considerable del éxito de las Uniones se debió a que no respondían a ninguna estructura estatal y ni siquiera eran controladas por la ANAP. No obstante, las UMCA-E encontraron numerosas puertas cerradas debido al desconocimiento por numerosas organizaciones empresariales e instituciones de esta experimental cooperativa de comercialización.

3 Excepto en Consolación del Sur, donde la producción de tabaco decreció $20 \%$ en la etap. de 1981-1984. 
Estructuralmente disponía de tres niveles:

El órgano superior de dirección de la Unión era la Asamblea de Representantes de los cooperativistas y de los campesinos individuales. Los presidentes de las CPA y CCS integrantes participaban por derecho propio y a nombre de los cooperativistas que las integraban. Aquí se aprobaban las líneas de la actividad económica y los planes de producción, entre otros aspectos.

Los presidentes de las CPA y de las CCS integraban el Consejo de Dirección junto a los responsables administrativos de la UMCA. Mensualmente tomaban decisiones dirigidas a asegurar el cumplimiento de las metas productivas con el Estado. Además, rendía cuenta periódicamente de su gestión ante los productores y ante los propios trabajadores de su aparato administrativo. Esta participación de los cooperativistas en las decisiones de la UMCA no tuvo igual manifestación en todos los municipios del país.

El nivel de base para la participación estaba formado por los ingenieros, técnicos, especialistas, obreros, oficinistas y asesores del aparato administrativo de la Unión, los cuales ejecutaban los abastecimientos, los servicios productivos y la asistencia técnica al sector no estatal del territorio. La dirección del aparato administrativo de las Unión rendía cuenta de su gestión periódicamente ante estos trabajadores.

La búsqueda desesperada de la rentabilidad llevó a algunas UMCA-E a aplicar recargos a las mercancías, lo cual repercutió negativamente en los costos de producción de algunos cultivos no muy rentables. La falta de gestión y el paternalismo hacia los campesinos condujeron a las Uniones a transformar cuentas por cobrar en cuentas incobrables. Algunas Uniones funcionaron mal de forma general y carecían de buena dirección, tanto que según informe del MINAGRI en el año 1986 sólo cuatro de ellas tenían favorables saldos financieros.

En la segunda mitad de la década de los 80 fue interrumpido este experimento de las UMCA en el marco de la política de rectificación de errores y tendencias negativas. No obstante su interrupción por no haber disfrutado de condiciones mínimas para su buen funcionamiento, no puede negarse el aporte que hicieron a la economía nacional. Entre estos aportes destacan: la mejoría en el servicio de abastecimientos en recursos productivos y en recursos de destino social; aplicaron diversas soluciones para economizar a favor de los productores en medios de producción, recursos laborales y monetarios; crearon reservas materiales de insumos; fungieron como una alternativa viable para la organización de los abastecimientos al sector cooperativo y campesino.

Sobre esta institución LABRADOR (1998, p. 62 y 63) plantea: «La experiencia de las UMCA-E demostró que la descentralización y organiza- 
ción de la cooperación entre empresas estatales y cooperativas es una vía correcta siempre que se aleje de la ausencia de control y regulación estatal y se apart. de la dualidad político-administrativa que se introdujo al fusionarse la dirección de la ANAP con la de las UMCA-E, violándose lo previsto en el reglamento original».

Las insuficiencias presentadas en el funcionamiento de las UMCA-E llevaron a la puesta en práctica de un nuevo sistema para la interrelación de las empresas y entidades del sector agrícola que arreció la centralización y el control estatal sobre el cooperativo. A tal fin se crearon los Consejos de Cooperación Agropecuaria (CONCA) y los Consejos de Cooperación Agroindustrial (CONCAI), concebidos como elementos necesarios para garantizar la coordinación, integración y fiscalización de las actividades a nivel local y desarrollar el proceso de cooperativización.

Los CONCA y los CONCAI aportaron al funcionamiento de las cooperativas mayor disciplina económica, fortalecimiento orgánico, incremento de los volúmenes de producción, aumento de la calificación de la fuerza de trabajo y perfeccionamiento de los mecanismos estatales de atención al sector. Sin embargo, la prevalencia, control y paternalismo de las instituciones estatales sobre las cooperativas que los integraron, alejaron su esencia de la identidad del movimiento. Por tanto, no sería apropiado considerarlos como estructuras superiores de integración del sector cooperativo, que - a criterio de los autores- estuvo directamente vinculado a su desaparición.

\section{III.2. Contexto actual y perspectivas}

El contexto cubano actual se caracteriza por el desarrollo de un proceso de actualización del modelo económico. Este reordenamiento responde, entre otros factores, a que la economía cubana en los últimos años se ha caracterizado por la baja eficiencia, descapitalización de la base productiva y de la infraestructura. En cuanto al funcionamiento de la economía, a partir del año 2003, se produjo una elevada centralización de los mecanismos de asignación y utilización de las divisas. Desde el año 2005 se evidenciaron las limitaciones para enfrentar el déficit de la cuenta financiera de la balanza de pagos, las retenciones bancarias de transferencias al exterior y el elevado monto de los vencimientos de la deuda. Todo ello significó una gran tensión en el manejo de la economía, situación que debe ser revertida mediante la apertura a nuevas alternativas.

La promoción de la cooperativa puede ser muy útil para conseguir el objetivo de mantener las conquistas sociales y tener también eficien- 
cia y sustentabilidad económica. Una cooperativa que funcione correctamente no presenta óbice para que sea tan o más eficiente que una empresa privada o estatal. Además cuenta a su favor con todos los beneficios que representa para sus miembros y la disposición para asumir compromisos sociales.

Criterios bien calificados como el de RIVERA (2012) apuestan con optimismo a que:

«En Cuba tenemos condiciones para promover cooperativas mejor que en otros países, porque el Estado es dueño de la mayoría de los medios de producción. Por otro lado, tenemos la ventaja de que nuestra población tiene índices de educación formal mayores que en otros lugares, aunque hay un desconocimiento y una confusión grandísimos sobre qué cosa es una cooperativa porque los referentes principales han sido las CPAs o las UBPCs. Pero las condiciones son favorables.» (p. 44)

Por ello, en los Lineamientos de la Política Económica y Social del Partido y la Revolución, aprobados por el VI Congreso del Partido Comunista de Cuba, que regirán durante los próximos años las transformaciones del país para perfeccionar su modelo socioeconómico, resaltan bajo el título de «LAS COOPERATIVAS» los numerales del 25 al 29. Así se destaca que estas entidades formarán part. de un nuevo «MODELO DE GESTIÓN ECONÓMICA» que reconocerá y estimulará otras formas empresariales para la producción de bienes o la prestación de servicios en disímiles esferas de la economía, a fin de elevar la productividad del trabajo. Específicamente, el lineamiento número 29 concibe como una alternativa para ese mejoramiento de las condiciones económico-sociales a las cooperativas de segundo grado.

Pero, como se ha apuntado en otras ocasiones (HeRnández \& RodríGUEZ, 2011), el ordenamiento jurídico cubano presenta una serie de limitaciones que dificultan el desarrollo integral del cooperativismo, a las que habría de sumarse la ausencia de un marco regulatorio de las cooperativas de segundo grado. No obstante, lo anterior tampoco significa que la interpretación de la normativa vigente tenga que ser llevada a puntos extremos en los que deba admitirse que su instrumentación sería contraria a la esencia del modelo constitucional concebido.

En tal sentido, si bien el artículo 23 de la Constitución cubana no se incorporó en la reforma constitucional de 1992 con esta finalidad, su contenido reconoce la propiedad de las sociedades y asociaciones económicas de forma genérica. Por tanto, no sería desatinado alegar dicho precepto para respaldar a priori la instrumentación legal de las cooperativas en diferentes sectores de la economía nacional más allá del agropecuario, amén de que su reconocimiento expreso en la Constitu- 
ción tan solo se perfila para el sector agropecuario de la economía. Y este análisis es válido tanto para las cooperativas primarias como para las de grado superior.

A fin de cuentas, con independencia de su tamaño y complejidad, habrían de funcionar sobre reglas tendentes a asegurar el control democrático y la participación económica de los asociados, lo que resulta consecuente con la «supresión de la explotación del hombre por el hombre» y con el principio de distribución socialista " de cada cual según su capacidad, a cada cual según su trabajo» ambos plasmados en el artículo 14 de la Constitución.

El legislador ordinario, hasta tanto se reforme la letra constitucional, para establecer los principios que informen, ordenen y promuevan las cooperativas durante esta fase experimental, debe encontrar límite y sustento en la interpretación de sus principales fundamentos. Sería un error entender que, al no aludirse expresamente a las cooperativas (no agropecuarias, de segundo o ulterior grado) en la letra del texto, su instrumentación legal vendría a contradecir el espíritu socialista del magno cuerp. jurídico.

Sin que se refiera en ninguno de los «POR CUANTO» la Constitución, al finalizar el año 2012 entró en vigor un paquete legal que contiene dos decretos-leyes del Consejo de Estado (No. 305 y 306, del 15 y 17 de noviembre de 2012), un Decreto del Consejo de Ministros (No. 309, del 28 de noviembre de 2012), una Resolución del Ministerio de Finanzas y Precios (No. 427/2012), y otra del de Economía y Planificación (No. 570/2012). Estos cuerpos jurídicos completan el marco regulatorio de las cooperativas no agropecuarias en Cuba.

Con amparo en esta normativa, para marzo del presente año sumaban 498 las cooperativas de trabajo aprobadas (mediante un riguroso proceso) y 224 las constituidas (mediante escritura notarial e inscripción en el Registro Mercantil previa autorización administrativa) en los sectores de gastronomía, construcción, trasporte, industria, alimentación, energía y servicios contables (MARTínez \& PUIG, 2014). En su mayoría se trata de actividades y estructuras que el Estado no ha gestionado de forma eficiente y de las que necesita irse desprendiendo para concentrarse en aquellas trascendentales para la economía (SÁNCHEZ, 2014).

Por su parte, el Decreto-Ley 305 «DE LAS COOPERATIVAS NO AGROPECUARIAS» fija, entre los principios que han de sustentar el actuar de estas figuras, la "colaboración y cooperación entre cooperativas y con otras entidades» [artículo 4, inciso g)]. Además, el artículo 5 , en su apartado primero admite la posibilidad de que se constituyan cooperativas de segundo grado. El tercer apartado del propio artículo 
copia - parcialmente - el concepto de cooperativas de segundo grado que ofrece el lineamiento 29 y que se pronuncia acerca de quiénes se asocian (dos o más cooperativas de primer grado) y cuáles son sus fines (organizar actividades complementarias afines o que agreguen valor a los productos y servicios de sus socios, o de realizar compras y ventas conjuntas, con vistas a lograr mayor eficiencia).

Ante la parca regulación de las cooperativas de cooperativas el mentado Decreto-Ley, en su Disposición Especial Cuarta, declara aplicable este cuerp. normativo a las cooperativas de segundo grado en aquellos términos en los que le sea pertinente. No obstante, no desconoce el legislador las incapacidades de dicha norma para ser exportada a una forma de organización superior y en su Disposición Final Sexta insta al Consejo de Ministros a dictar el Reglamento de las Cooperativas de Segundo Grado en un plazo de 360 días luego de la publicación del Decreto-Ley en la Gaceta Oficial (11 de diciembre de 2012). Pero, bien entrado el año 2014, el Reglamento de las cooperativas no agropecuarias de segundo grado aún no se aprueba.

La pausa en el proceso de reglamentación, aprobación y constitución de las cooperativas de segundo grado puede encontrar fundamento en los problemas que han estado confrontando las cooperativas no agropecuarias de base. Al observar la marcha del experimento en la provincia de Pinar del Río la prensa (MolinA, 2014 y SuÁrez \& GonZÁlEZ, 2014) ha señalado algunas dificultades que pudiéramos sistematizar como sigue:

- Afectaciones a la voluntariedad en la constitución, en tanto no llegó a unidades estatales cuyos colectivos expresaron su decisión expedita de acogerse a esta forma de gestión, sino que fueron escogidos a partir de los expedientes presentados a la dirección nacional y que de acuerdo con el formato y el cronograma de implementación clasificaron para la experiencia. Por ello no podemos asegurar que en todo caso, en los actuales «cooperativistas» no agropecuarios exista la conciencia suficiente para ofrecer eficacia al principio de "voluntariedad» que debe sustentar a estas entidades según se establece en el inciso a) del artículo 4 del DL305/2012; podrían estar movidos más bien por la necesidad de no perder su empleo. Debe trabajarse más para generar la percepción sobre las necesidades, ventajas y responsabilidades que implica la asimilación de esta nueva forma de gestión.

- Resultado de obstáculos burocráticos que subsisten, de la mala planificación y de la baja productividad que caracteriza a la economía, no existe un sistema de abastecimiento en correspon- 
dencia con las demandas de las cooperativas que les permita obtener, a precios mayoristas (en ocasiones tampoco a precios minoristas), los indispensables insumos y las materias primas que necesitan.

- La no existencia de este mercado trae consigo el incremento de precios de ventas en las unidades, lo que tiene un impacto negativo sobre los clientes. De esta forma resulta difícil conjugar, tal como demanda el DL305/2012 [inciso f) del artículo 4], la responsabilidad social y el bienestar de los socios y sus familiares, principios en que se sustentan estas entidades: "Los planes de las cooperativas tienen como objetivo contribuir al desarrollo económico y social sostenible de la nación, proteger el medio ambiente [y] desarrollar sus actividades sin ánimo especulativo...».

Las cooperativas de segundo grado son la extensión organizada del movimiento cooperativo. Dado su superior tamaño, es innegable que poseen más poder y permiten al sector hacerse oír con mayor potencia ante la sociedad y ante la Administración Pública. De esta forma los intereses del cooperativismo podrían presentar un respaldo más sólido.

Sin embargo, no hay dudas de que las cooperativas de segundo o ulterior grado implican un salto cualitativo en la organización del movimiento en cualquier contexto que exige, para ser efectivo, de una solidez suficiente en la base. Esta fuerza ha de expresarse -entre otros elementos - en el número de cooperativas de primer grado, en la consolidación de sus indicadores socioeconómicos, en la experiencia teórico-práctica de sus miembros y en la existencia de una plataforma legal apropiada que también organice un ambiente institucional coherente con la identidad del movimiento.

Sobre estos presupuestos, quizás sea prudente instrumentar los ensayos de este tip. en el sector agropecuario, donde existen antecedentes que los respaldan y donde el movimiento cooperativo nacional - con sus luces y sombras - está más consolidado. Lamentablemente en este sector de la economía aún no existe nada legislado al respecto.

Por tanto, vale la pena analizar con suspicacia las condiciones objetivas y subjetivas en Cuba para avanzar hacia la creación de cooperativas de segundo o ulterior grado. A criterio de los autores aún no es el momento para dar este paso, lo cual no niega que un futuro pudiera resultar -incluso- necesario.

No en balde se insiste desde la dirección política del país en que:

«Se trata de un proceso experimental, y aunque se avanza, su propósito fundamental es el de ir detectando posibles fallos y corre- 
girlos. De ahí la necesidad de que los organismos rectores de las actividades donde se desempeñan estas actividades, así como los gobiernos provinciales y municipales, chequen el desempeño de ellas y saquen las mejores experiencias... No podemos apresurarnos en la aprobación constante de estas cooperativas. Iremos al ritmo que corresponda. (General de Ejército Raúl Castro Ruz, Presidente de los Consejos de Estado y de Ministros en Reunión del Consejo de Ministros del 1 de marzo de 2014, citado por Suárez \& González, 2014).»

\section{Conclusiones}

1. Las cooperativas de segundo o ulterior grado constituyen una entidad con naturaleza cooperativa que ostentan personalidad jurídica propia e independiente a la de sus socios, cuyo surgimiento responde — principalmente- a la necesidad de desarrollo económico de sus miembros y a la intercooperación propia del movimiento. Estas formas - por excelencia- para la integración cooperativa ofrecen una serie de ventajas a sus miembros (respeto a los principios cooperativos; las sociedades que se integran mantienen su autonomía y se benefician en aspectos relativos a la administración, la producción y la comercialización; etc.) que se aderezan con algunas dificultades (disminución de la capacidad de decisión; soporte de costes extras; adopción de una democracia delegada; necesidad de personal cualificado - no siempre disponible- para una gestión más compleja; resistencia subjetiva al cambio; etc.). El éxito del proceso de integración se encuentra íntimamente relacionado con la capacitación de los implicados y las circunstancias que los rodean.

2. El contexto cubano actual se caracteriza por el desarrollo de un proceso de actualización del modelo económico, donde se concibe como una alternativa para el mejoramiento de las condiciones económico-sociales del país a las cooperativas de segundo grado en sectores diferentes al agropecuario. Sin embargo, al observar las carencias del ordenamiento jurídico cubano en esta materia y los problemas que han estado confrontando las cooperativas no agropecuarias de base, es criterio de los autores que aún no es el momento para dar este paso. Quizás sea prudente instrumentar los ensayos de este tip. en el sector agropecuario, donde existen antecedentes que los respaldan. 


\section{Bibliografía}

\section{TEXTOS IMPRESOS}

AIZEGA, J.H. \& VALIÑANI, E. (2003): Las cooperativas de segundo grado, grup. cooperativo y otras formas de colaboración económica, Revista de Estudios Cooperativos (REVESCO), n. ${ }^{\circ} 79$.

ALFONSO, R. (2000): La integración cooperativa y sus técnicas de realización: La cooperativa de segundo grado, Editorial Tiran Lo Blanch, Valencia.

ARCAS, N. (2002): Contribución de las cooperativas agrarias de segundo grado a la orientación al mercado de sus cooperativas asociadas: efectos en el desempeño de la relación, Revista de Economía Pública, Social y Cooperativa, CIRIEC-España, n. ${ }^{\circ} 41$.

ARCAS, N., MUNERA, J. \& HERNÁNDEZ, M. (2002): Beneficios de las cooperativas agrarias de segundo grado: Contribución a los objetivos de sus socios», Revista de Estudios Cooperativos (REVESCO), n. ${ }^{\circ} 76$.

BRUQUE, S. \& MOYANO, J. (2006): Integración cooperativa y sistemas de información interorganizativos. Un análisis aplicado al cooperativismo oleícola, Revista de Economía Pública, Social y Cooperativa, CIRIEC-España, n. ${ }^{\circ} 54$.

CUENCA, A. (2000): Las cooperativas de segundo grado en la Ley 27/1999 de 16 de julio de cooperativas, Revista Jurídica de Economía Social y Cooperativa, CIRIEC - España, n. ${ }^{\circ} 11$.

FAJARDO, G. (2005): Intercooperación e integración cooperativa en la legislación española, en Cooperativismo Agrario y Desarrollo Rural (Congreso Internacional, 25 aniversario (EGEA), Editorial UPV, Valencia.

GADEA E., SACRITÁN F. \& VARGAS C. (2009): Régimen jurídico de la sociedad cooperativa del siglo XXI. Realidad actual y propuestas de reforma, Editorial Dykinson, Madrid.

GARCÍA, Y. (2013): Las cooperativas no agrícolas: una invitación a la reflexión y la acción, Revista Vitral, octubre-diciembre n. ${ }^{\circ} 112$, Pinar del Río.

GARrido, T., Puentes, R. \& Navarro, E. (2007): Fiscalidad de las sociedades cooperativas de segundo grado, Revista de Estudios Cooperativos (REVESCO), n. ${ }^{\circ} 91$.

GIRÓN, J. (1976): Derecho de sociedades, Tomo I: Part. General, Sociedades Colectivas y Comanditarias, Editorial G.T., Madrid.

HERNÁNDEZ, O. \& RodríGUEZ, O. (2011): La cooperativa en el ordenamiento jurídico cubano. Una aproximación crítica a la luz del actual proceso de perfeccionamiento del modelo económico en el país, Boletín de la Asociación Internacional de Derecho Cooperativo n. ${ }^{\circ} 45$, Universidad de Deusto, Bilbao.

KAPLAN DE DRIMER, A. y DRIMER, B. (1981): Las cooperativas. Fundamentos Historia - Doctrina, Editorial Intercoop. Buenos Aires.

LABRADOR, O. (1998): La eficiencia económica de las cooperativas tabacaleras en los marcos de la reestructuración de la economía cubana, Tesis en opción al grado de Doctor en Ciencias Económicas, Universidad de Pinar del Río. 
MARTÍNEZ, A. (1990): Análisis de la integración cooperativa, Universidad de Deusto, Bilbao.

MARTíNEZ, A. (2012): Sobre el principio de integración entre cooperativas en la actualidad, Boletín de la Asociación Internacional de Derecho Cooperativo n. ${ }^{\circ} 46$, Universidad de Deusto, Bilbao.

MARTínEZ, L. \& PUiG, Y. (2014, Marzo 3): Insta Raúl al análisis crítico sobre la marcha de la implementación, Periódico Trabajadores, La Habana, pp.4 y 5.

MCCORMACK, M. C. (2006): Temas de Derecho Agrario, Editorial Félix Varela, La Habana.

MELIÁ, E. (2003): «Cooperativas de segundo grado versus fusión. Ventajas e inconveniente Integración empresarial, cooperativas y economía social: posibilidades, ventajas e inconvenientes, II Coloquio Ibérico de cooperativismo y economía social, Valencia 13 y 14 de marzo, Universidad de Valencia.

MOLINA, Y. (2014, Enero 10): Cooperativismo vs. Accesibilidad, Periódico Guerrillero, Pinar del Río, pp.4 y 5.

MONTERO, A. (1991): Cooperativismo Agrario de Segundo Grado, Ministerio de Agricultura, Pesca y Alimentación, Madrid.

MONTERO, A. (1999): El cooperativismo agroalimentario y formas de integración, Ministerio de Agricultura, Pesca y Alimentación, Madrid.

MORENO, R. (2001): "Las empresas de participación aseguradoras ante la cooperación o concentración empresarial», Revista de Estudios Cooperativos (REVESCO), n. ${ }^{\circ} 73$.

MOZAS, A. \& BERNAL, E. (2004): «Integración cooperativa y TIC's: presente y futuro», Revista de Economía Pública, Social y Cooperativa, CIRIEC-España, n. ${ }^{\circ} 49$.

PARRAS DE MÁS, S. (1974): La integración de la empresa cooperativa (Evolución de los principios cooperativos), Editorial de Derecho Financiero, Madrid.

PEISO, A. (2012): «La Cooperativa. Bases para su Legislación en Cuba», Editorial Ciencias Sociales, La Habana.

RIVERA, C.A. (2012): «Cooperativismo, Gestión y Desarrollo Social», Centro de Estudio sobre Desarrollo Cooperativo y Comunitario, Universidad de Pinar del Río.

RODRÍGUEZ, O. (2012): La cooperativa como figura jurídica. Perspectivas constitucionales en Cuba para su aprovechamiento en otros sectores de la economía nacional además del agropecuario, Editorial Dykinson SL., Madrid.

SÁNCHEZ, O. (2014, Enero 10): «La materialización de una estrategia», Periódico Granma (Suplemento Especial), La Habana, pp.2-4.

SANCHIS, J. (1995): Análisis estratégico de la empresa cooperativa, Tirant lo Blanch, Valencia.

SENISE, O. (2003): Integración cooperativa y orientación al mercado: el caso de las cooperativas oleícolas andaluzas, Tesis Doctoral, Universidad de Jaén.

SENISE, O. \& PARRAS, M. (2005): «Cooperación entre empresas como proceso dinámico y su influencia en los resultados: una aproximación desde el cooperativismo oleícola andaluz», Revista de Economía Pública, Social y Cooperativa, CIRIEC-España, n. 51. 
SUÁREZ, R. \& GONZÁLEZ, E. (2014, Febrero 7): Cooperativas no agropecuarias. Luces y Sombras, Periódico Granma, La Habana, p. 12.

VARGAS, A. (1995): Claves para el desarrollo del cooperativismo agrario. Concentración empresarial y asociacionismo, Universidad de Huelva.

VICENT, F. (1991): Compendio crítico de Derecho Mercantil, tomo I, Editorial José M. Bosch, Barcelona.

\section{TEXTOS Digitales}

- ACI: «Declaración Sobre la Identidad Cooperativa», Manchester, septiembre de 1995, reproducida y comentada por El hogar obrero: Cooperativa de consumo, edificación y crédito LTDA en http://www.elhogarobrero1905.org.ar, consultada el 17 de diciembre de 2012.

- «Cuba: realidad y perspectiva del cooperativismo». Entrevista a Camila Piñeiro Harnecker, investigadora del Centro de Estudios de la Economía Cubana, publicada el 29 de enero de 2013 en http://www.centrocultural. coop/blogs/cooperativismo/2013/01/29/cuba-realidad-y-perspectivas-delcooperativismo/, consultado el 12 de abril de 2013.

- CRUz, J. (2012): "Cooperativas de segundo grado en Cuba (experiencia de 1982/86)», ponencia presentada en el VIII Congreso Internacional de Derecho Agrario, La Habana, 24, 25 y 26 de abril de 2012.

- Central de Trabajadores de Cuba (2010): «Pronunciamiento de la Central de Trabajadores de Cuba», 13 de septiembre en www.cubadebate.cu/noticias/2010/09/13/reducira-cuba-medio-millon-de-plazas-en-elsector-estatal, consultado el 2 de octubre 2012.

- Puentes, R. (2010): "Las cooperativas de segundo grado como forma de integración: especial referencia al efecto impositivo», Tesis en opción al grado de Doctor en Ciencias Económicas en http://www.eumed.net/tesisdoctorales/2008/rpp/lascooperativasdesegundogradocomoformadeintegracionindicedecuadros. htm consultado en febrero de 2013.

- Puentes, R., Antequera, J.M. \& Velazco, M. (2010): «las sociedades cooperativas de segundo grado y su relación con socios» en http://www.ucm.es/ info/revesco/txt/revescon93.4raquelpuentesmariadelcarvelasco,josemiguela ntequera.htm, consultado en febrero de 2013.

- VI Congreso del Partido Comunista de Cuba: Lineamientos de la Política Económica y Social del Partido y la Revolución, 18 de abril de 2011, recuperado de http://www.cubadebate.cuwp-contentuploads201105folleto-lineamientos-vi-cong.pdf, consultados el 24 de abril de 2011.

Legislación:

- Constitución de la República de Cuba de 24 de febrero de 1976,reformada en los años 1992 y 2002, Ministerio de Justicia, La Habana, 2004.

- Decreto Ley 305 «De las Cooperativas No Agropecuarias» publicado en la Gaceta Oficial No. 53, Extraordinaria, Año CX, martes 11 de diciembre de 2012. 
- Decreto Ley 306 «Del Régimen Especial de Seguridad Social de los Socios de las Cooperativas No Agropecuarias» publicado en la Gaceta Oficial No. 53, Extraordinaria, Año CX, martes 11 de diciembre de 2012.

- Decreto 309 «Reglamento de las cooperativas no Agropecuarias» publicado en la Gaceta Oficial No. 53, Extraordinaria, Año CX, martes 11 de diciembre de 2012. 